EPJ Web of Conferences 16, 04004 (2011)

DOI: $10.1051 /$ epjconf/20111604004

(C) Owned by the authors, published by EDP Sciences, 2011

\title{
Testing substellar models with dynamical mass measurements
}

\author{
T.J. Dupuy ${ }^{1, a}$, M.C. Liu ${ }^{1}$ and M.J. Ireland ${ }^{2}$ \\ ${ }^{1}$ Institute for Astronomy, University of Hawai'i, 2680 Woodlawn Drive, Honolulu, HI 96822, \\ USA \\ ${ }^{2}$ School of Physics, University of Sydney, NSW 2006, Australia
}

\begin{abstract}
We have been using Keck laser guide star adaptive optics to monitor the orbits of ultracool binaries, providing dynamical masses at lower luminosities and temperatures than previously available and enabling strong tests of theoretical models. We have identified three specific problems with theory: (1) We find that model color-magnitude diagrams cannot be reliably used to infer masses as they do not accurately reproduce the colors of ultracool dwarfs of known mass. (2) Effective temperatures inferred from evolutionary model radii are typically inconsistent with temperatures derived from fitting atmospheric models to observed spectra by 100-300 K. (3) For the only known pair of field brown dwarfs with a precise mass $(3 \%)$ and age determination $(\approx 25 \%)$, the measured luminosities are $\sim 2-3 \times$ higher than predicted by model cooling rates (i.e., masses inferred from $L_{\text {bol }}$ and age are $20-30 \%$ larger than measured). To make progress in understanding the observed discrepancies, more mass measurements spanning a wide range of luminosity, temperature, and age are needed, along with more accurate age determinations (e.g., via asteroseismology) for primary stars with brown dwarf binary companions. Also, resolved optical and infrared spectroscopy are needed to measure lithium depletion and to characterize the atmospheres of binary components in order to better assess model deficiencies.
\end{abstract}

\section{INTRODUCTION}

Detailed theoretical models of stars, developed and observationally tested over the last century, now underlie most of modern astronomy. However, the lowest mass stars $\left(M_{\star} \lesssim 0.1 M_{\odot}\right)$ are sufficiently cool $\left(T_{\text {eff }} \lesssim 3000 \mathrm{~K}\right)$ that the standard, well-tested stellar models are not appropriate (e.g., due to dust formation in the photosphere). Objects below the hydrogen-fusing mass limit can cool to even lower temperatures as they have no sustained source of internal energy generation. At temperatures below $\sim 2000 \mathrm{~K}$, dust and $\mathrm{H}_{2} \mathrm{O}$ are the major sources of opacity in the photosphere, and below $\sim 1400 \mathrm{~K}$ methane absorption becomes important. Over the last decade, new theory has been developed to describe such low-temperature objects, which encompass brown dwarfs and gas-giant planets (e.g., [1, 2, 4$6,13])$. These models now form the basis of our understanding of all low-mass gaseous objects, from stars at the bottom of the main sequence to extrasolar giant planets. Thus, rigorously testing them is vitally important.

Dynamical masses for "ultracool" visual binaries (i.e., those with spectral types later than $\sim$ M7) are central to this effort, but such measurements have previously been impeded by observational limitations. Most ultracool binaries were discovered $\sim 5$ to 10 years ago, and the shortest estimated orbital periods are long ( $\gtrsim 10-30$ years). The corresponding physical separations $(\sim 1-3 \mathrm{AU})$ result in very small angular separations $(\lesssim 0.2 \mathrm{arcsec})$ that can only be resolved using the Hubble Space Telescope (HST) or ground-based adaptive optics (AO). Direct distance measurements via trigonometric parallax are also essential as they are needed to convert the observed angular scale of the orbit into physical units and

ae-mail: tdupuy@ifa.hawaii.edu

This is an Open Access article distributed under the terms of the Creative Commons Attribution-Noncommercial License 3.0, which permits unrestricted use, distribution, and reproduction in any noncommercial medium, provided the original work is properly cited. 

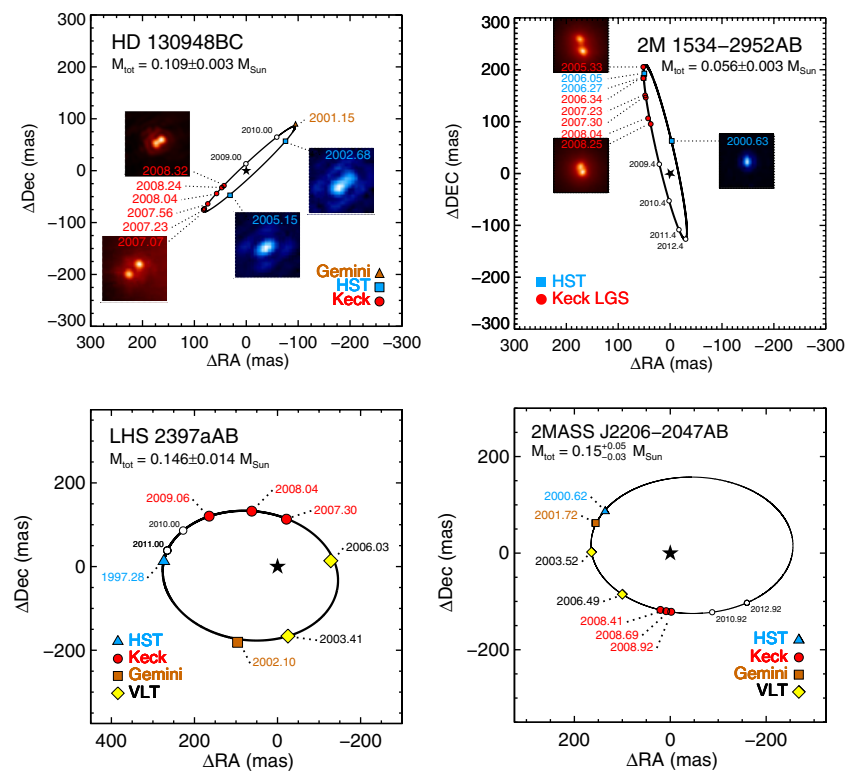

Figure 1. Our Keck LGS AO data combined with discovery and archival data from 5 to 10 years ago enables precise orbit determinations for ultracool binaries. Top left: HD 130948B and C are companions to a young solar analog $(\mathrm{G} 2 \mathrm{~V}, 0.8 \pm 0.2 \mathrm{Gyr})$, making them the first field brown dwarfs (L4+L4) with a well-determined age and masses $\left(M_{\mathrm{tot}}=0.109 \pm 0.003 M_{\odot} ;[10]\right)$. Top right: $2 \mathrm{MASS} \mathrm{J} 1534-2952 \mathrm{AB}$ is the first T dwarf binary with a dynamical mass $\left(\mathrm{T} 5+\mathrm{T} 5.5, M_{\mathrm{tot}}=0.056 \pm 0.003 M_{\odot}\right.$ ), revealing inconsistencies between the atmospheric model-derived temperatures, evolutionary model H-R diagram, and measured mass [14]. Bottom left: LHS 2397aAB (M8+L7, $M_{\text {tot }}=0.146 \pm 0.014 M_{\odot}$ ) is the first dynamical mass benchmark at the $\mathrm{L} / \mathrm{T}$ transition, showing consistency between temperatures estimated from atmospheric and evolutionary models and supporting the idea that the temperature of the L/T transition is surface gravity dependent [11]. Bottom right: 2MASS J2206-2047AB $(\mathrm{M} 8+\mathrm{M} 8)$ is a pair of stars at the bottom of the main sequence that have $J$-band colors $0.2-0.3$ mag redder than predicted by evolutionary model tracks for objects of their measured masses [9]. This implies that masses and/or ages inferred from model color-magnitude diagrams will be in error for such objects.

equally importantly to provide a direct measurement of the luminosity. As a result, previous to our work only three ultracool binaries had measured dynamical masses $([3,8,20])$, and these were all relatively warm ( $\gtrsim 2100 \mathrm{~K})$.

\section{DYNAMICAL MASSES OF ULTRACOOL BINARIES}

We have been using Keck laser guide star (LGS) AO direct imaging and aperture masking to monitor the orbits of ultracool binaries, enabling dynamical mass measurements for the lowest mass (30$\left.75 M_{\text {Jup }}\right)$, lowest temperature $(1000-2800 \mathrm{~K})$, lowest luminosity $\left(10^{-4}\right.$ to $\left.10^{-5} L_{\odot}\right)$ objects known. This has allowed models to be tested in the unexplored area of parameter space shared by brown dwarfs and extrasolar giant planets. Keck LGS AO delivers diffraction-limited imaging in the infrared for targets over most of the sky $(\sim 0.05 "$ FWHM; $\sim 4 \times$ sharper than HST), so it is ideally suited for resolving short-period ultracool binaries. By performing a detailed analysis of these high-resolution images and accounting for small astrometric shifts due to differential atmospheric refraction, we routinely achieve sub-milliarcsecond astrometric accuracy and $\sim 200 \mu$ as for our best data. Such high quality astrometry has allowed us to precisely measure binary orbits, with the error in the resulting masses (typically 3$10 \%$ ) dominated by the uncertainty in the distance (Figure 1). 

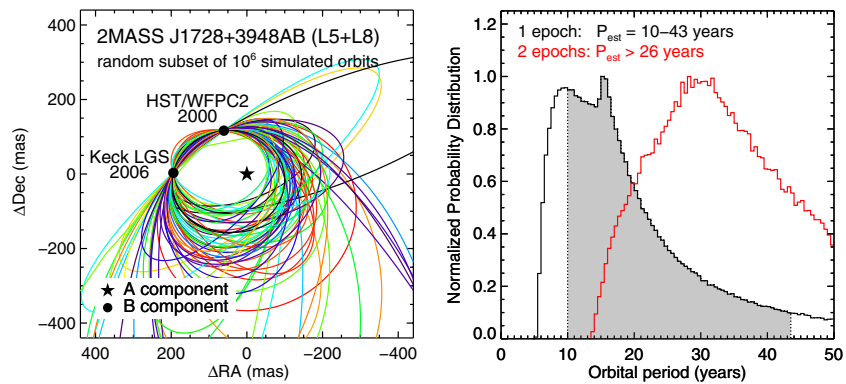

Figure 2. We have developed a novel Monte Carlo technique to determine the orbital period probability distribution from orbital motion observed between only two epochs. Left: For the ultracool binary 2MASS J1728+3948AB, the relative positions of the $\mathrm{A}$ and $\mathrm{B}$ components are shown at the discovery epoch and $\sim 6$ years later from our Keck LGS AO program (filled symbols). Using the approach described in the text, we randomly drew orbits that pass through the two observed positions at the appropriate epochs (multi-colored lines). Right: The orbital period distribution of the randomly drawn orbits (red) compared to the estimated period distribution using only the discovery epoch (black), following the method of Torres [19], with $\pm 1 \sigma$ confidence limits shaded gray. Although the two-epoch distribution appears at face value to be broader and thus less precise, it is actually strongly preferred as it is free of the somewhat arbitrary assumptions required by the single-epoch estimate (i.e., a uniform eccentricity distribution between $0<e<1$ and a total mass of $0.135 M_{\odot}$ ). In the case of 2MASS J1728+3948AB, we found that the orbital period is on the longer side ( $P>26$ years, $68.3 \%$ c.1.) of the original estimate (10-43 years, $68.3 \%$ c.l.), reducing its priority in our orbital monitoring program.

We have also undertaken a substantial amount of supplementary analysis to enable these mass measurements:

(1) We have re-analyzed archival HST images from 5 to 10 years ago, improving astrometric errors by a factor of 2 to 8 compared to published values, and this has proved critical for accurate orbit determination (e.g., [11, 14]).

(2) We have developed a novel Monte Carlo technique to determine the orbital period probability distribution from motion observed between discovery and our first Keck data obtained $\gtrsim 5$ 10 years later (Figure 2). This has enabled us to accurately gauge target priorities and thus measure dynamical masses faster with a limited amount of telescope time. The orbital period, and thus the monitoring priority, of a visual binary is very uncertain from a single observation. In order to estimate the period probability distribution from a single observation, one must assume both a total mass and eccentricity probability distribution, and even with these assumptions the $\pm 1 \sigma$ confidence limits span a factor of $\sim 4$ in orbital period [19]. Our method utilizes the two positions and two times of two observations taken several years apart to eliminate 4 of the 7 orbital parameters (the two "geometrical" parameters semimajor axis and eccentricity; and the two "time" parameters period and time of periastron passage). This leaves only 3 parameters (inclination, argument of periastron, and position angle of the ascending node), which are just viewing angles that we conservatively assume to be distributed randomly according to appropriate distributions. We use a Monte Carlo approach that results in an ensemble of orbits that pass through the two observed positions at the observed epochs. The distribution of periods of these orbits is the period probability distribution. This method can, but does not necessarily, result in a narrower range of orbital periods; however, this method always results in a more accurate estimate of the period because it does not require an assumption about the eccentricity or total mass.

(3) Finally, and most importantly, we have been conducting an infrared parallax program at the Canada France Hawaii Telescope (CFHT; Figure 3). Precisely measured distances are critical for dynamical masses from visual binaries given the strong dependence of $M_{\text {tot }} \propto d^{3}$. Only about 1 


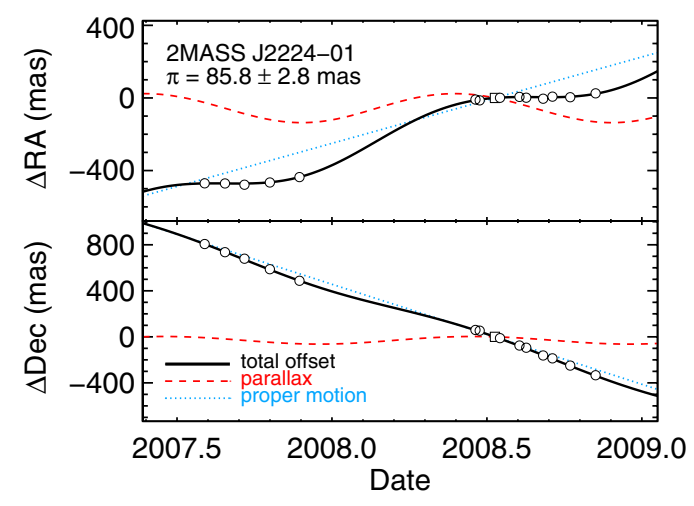

Figure 3. Analysis of data from our infrared parallax program at CFHT demonstrates the capability to measure precise distances to visual binaries in our Keck LGS AO sample, which is critical as the derived dynamical mass depends strongly on the distance $\left(M_{\text {tot }} \propto d^{3}\right)$. Our parallaxes of "control" objects that have published parallaxes, such as the object shown here, agree well with published results. Our astrometric measurements are shown as open circles (error bars are smaller than the plotting symbols) along with the best-fit astrometric solution (solid black) which includes parallax (red dashed) and proper motion (blue dotted) components.

in 4 of the shortest period ultracool binaries have previously published parallaxes, so our program targeting $\sim 30$ binaries enables a greatly expanded sample of masses.

\section{TESTING SUBSTELLAR MODELS}

Our Keck program has been yielding dynamical masses with the needed precision (3-10\%) to perform fundamental tests of theory (Figure 1). To date, we have identified three specific problems with substellar models:

(1) We find inconsistencies between predicted near-infrared colors and those observed for field objects of known mass over a wide range of spectral types ([9-11, 14]). For example, the M8+M8 components of 2MASS J2206- 2047AB and L4+L4 components of HD 130948BC appear to be $\approx 0.2-0.4$ mag redder than model tracks, while the T5+T5.5 components of 2MASS J1534-2952AB appear to be $\approx 0.2-0.4$ mag bluer. This is likely due to imperfect modeling of dust for the late-M and $\mathrm{L}$ dwarfs and incomplete methane line lists for the $\mathrm{T}$ dwarfs. Thus, masses and/or ages inferred from theoretical color-magnitude diagrams should be treated with caution.

(2) If theoretically predicted radii are correct, we have found that temperatures derived from atmospheric models are systematically in error $([9,10,14])$. This points either to inaccurate theoretical radii or to incomplete modeling of such low-temperature atmospheres. The one object for which this is not the case is LHS $2397 \mathrm{aB}$, currently the only mass benchmark at the L/T transition [11]. This is surprising as existing atmospheric models should not be appropriate for such transitional objects as their predictions are only valid in the limiting cases of maximal dust (Dusty, [6]) and the total absence of dust (COND, [2]). Thus, in the case of LHS 2397aB it is likely that large systematic errors cancel out to produce apparent agreement.

(3) For the only system with both a known mass and age, we have found the measured luminosities of the individual components to be $\sim 2-3 \times$ higher than predicted (Figure 4 ; [10]). This would imply that model-derived masses are significantly over estimated by $\sim 20-30 \%$. For example, an error of this magnitude is claimed to be needed to make the directly imaged extrasolar planets around HR 8799 dynamically stable as their model-derived masses seem to be $\sim 30 \%$ too high 


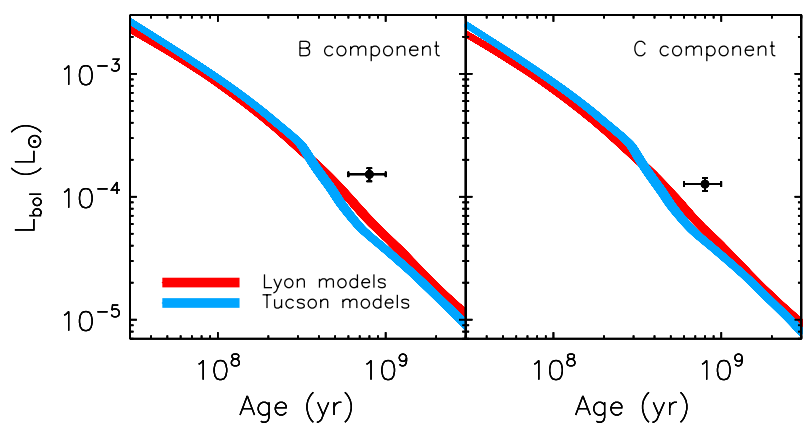

Figure 4. We have tested substellar cooling rates by measuring the masses and luminosities of HD 130948B and C, brown dwarfs with a well-determined age of $0.8 \pm 0.2 \mathrm{Gyr}$ from the primary star HD 130948A [10]. Surprisingly, they are $\sim 2-3 \times$ more luminous than evolutionary models predict. Two independent sets of theoretical luminosity tracks are shown as colored isomass lines, where the thickness corresponds to the uncertainty in the measured mass. Such a systematic error would imply that model-inferred masses are over estimated by $\sim 20-30 \%$ in the usual case where only the luminosity and age are known (e.g., for directly imaged extrasolar planets and determinations of cluster initial mass functions). The other possibility is that the age of HD 130948A is inaccurately estimated despite using the latest gyrochronology calibrations [15]. This can be resolved by obtaining a better age estimate (e.g., from asteroseismology) and measuring masses and ages for more such systems.

[12]. However, we emphasize that this apparent over-luminosity is based on a single system whose age is estimated from the primary star HD 130948A. While this young solar analog is fortuitously amenable to multiple age-dating techniques using stellar rotation, chromospheric and $\mathrm{x}$-ray activity, and isochrone fitting, precise age estimation is notoriously difficult for an arbitrary field star. Thus, refining the age estimate for HD 130948A (e.g., via asteroseismology) is essential. Also, more dynamical masses for the substellar binaries in triple systems with stars of known age (e.g., $\epsilon$ Ind Bab, Gl 417BC, GJ 1001BC) are critically needed to address this potential problem with model cooling rates.

\section{FUTURE WORK}

To make progress in understanding the problems we have found with substellar models, we are in the process of developing a larger sample of masses spanning a wide range in temperature, mass, and age. For example, more mass measurements should determine whether the observed over-luminosity of brown dwarfs (Figure 4) persists for different surface temperatures. If so, this would point to a problem with the interior structure model (e.g., convection) rather than a surface effect (e.g., magnetic fields).

Resolved spectroscopy is also needed to enable detailed characterization of the individual components' temperatures, surface gravities, and abundances. Binaries with known masses can provide stronger tests of atmospheric models than field brown dwarfs of unknown age or mass. Such measurements will be able to assess poorly understood atmospheric effects such as dust formation and sedimentation (e.g., [1]) and vertical mixing that can drive nonequilibrium chemistry (e.g., [16]).

In addition, optical spectra from HST/STIS will enable measurements of lithium absorption at $6708 \AA$ for binaries that we have shown are very close to the theoretically predicted lithium burning limit at $\approx 60 M_{\text {Jup }}$, providing a novel test of substellar interior models $([10,11])$. As shown in Figure 5, independent groups make different predictions for the mass limit of lithium depletion. Binaries close to this limit offer the chance to empirically determine the lithium boundary for the first time, with a precision comparable to our dynamical mass measurements $(\sim 3 \%)$. The theoretically predicted lithium boundary has never been directly tested in such a manner, even though it has been widely used, for example, to determine the canonical ages for young clusters such as the Pleiades [18]. 


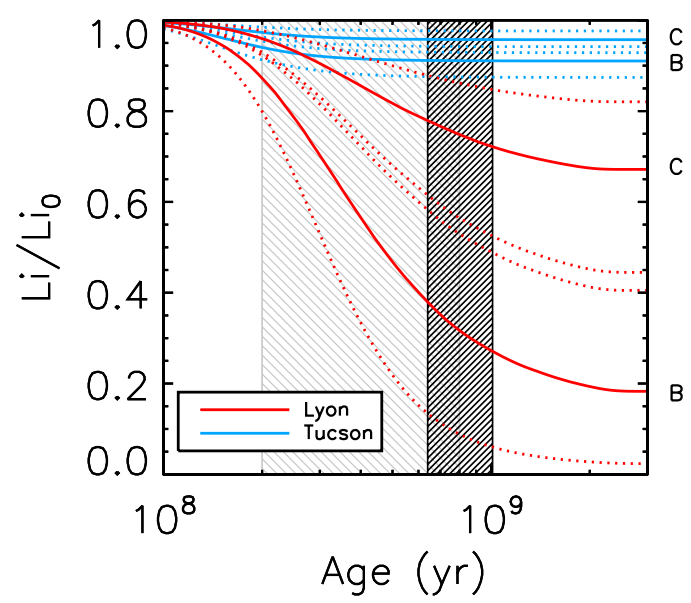

Figure 5. Lithium depletion as a function of age predicted by two independent sets of evolutionary models. The solid lines correspond to the individual masses of HD 130948B and C. These lines are bracketed by dotted lines that correspond to the $1 \sigma$ uncertainties in the individual masses. The ordinate is the fraction of initial lithium remaining. The hatched black box indicates the constraint from the age of the primary star HD 130948A estimated from gyrochronology (gray is the less precise but consistent estimate from chromospheric activity). The Tucson models predict that both of the binary components should be lithium-bearing, while the Lyon models predict that at least one of the two components should be massive enough to have destroyed its initial lithium. Thus, resolved optical spectroscopy of lithium absorption at $6708 \AA$ for this binary, only possible with HST/STIS, will provide the first test of the model-predicted lithium depletion boundary, which has been widely used, for example, to derive the canonical ages for young clusters such as the Pleiades [18].

Finally, a large sample of ultracool binary orbits will provide constraints on formation models for low-mass stars and brown dwarfs as the orbital parameters of binaries (e.g., semimajor axis and eccentricity) record the dynamical imprint of their formation process. For example, competing models predict very different eccentricity distributions for ultracool binaries: Stamatellos \& Whitworth predict high eccentricities $(e \gtrsim 0.5)$ for ultracool binaries formed via gravitational fragmentation in a massive circumstellar disk [17], while Bate predicts modest eccentricities $(e \lesssim 0.5)$ for ultracool binaries formed in a cluster environment [7].

\section{References}

[1] F. Allard, F., P. H. Hauschildt, D. R. Alexander, A. Tamanai, \& A. Schweitzer, ApJ 556, (2001) 357-372

[2] I. Baraffe, G. Chabrier, T. S. Barman, F. Allard, \& P. H. Hauschildt, A\&A 402, (2003) 701-712

[3] H. Bouy, et al., A\&A 423, (2004) 341-352

[4] A. Burrows, et al., ApJ 491, (1997) 856

[5] A. Burrows, D. Sudarsky, \& I. Hubeny, ApJ 640, (2006) 1063-1077

[6] G. Chabrier, I. Baraffe, F. Allard, \& P. Hauschildt, ApJ 542, (2000) 464-472

[7] M. R. Bate, MNRAS 392, (2009) 590-616

[8] C. Leinert, et al., A\&A 367, (2001) 183-188

[9] T. J. Dupuy, M. C. Liu, \& B. P. Bowler, ApJ 706, (2009a) 328-342

[10] T. J. Dupuy, M. C. Liu, \& M. J. Ireland, ApJ 692, (2009b) 729-752

[11] T. J. Dupuy, M. C. Liu, \& M. J. Ireland, ApJ 699, (2009c) 168-185

[12] D. C. Fabrycky \& R. A. Murray-Clay, ApJ (submitted; astro-ph/0812.0011)

[13] J. J. Fortney, K. Lodders, M. S. Marley, \& R. S. Freedman, ApJ 678, (2008) 1419-1435

[14] M. C. Liu, T. J. Dupuy, \& M. J. Ireland, ApJ 689, (2008) 436-460 
Research, Science and Technology of Brown Dwarfs and Exoplanets

[15] E. E. Mamajek \& L. A. Hillenbrand, ApJ 687, (2008) 1264-1293

[16] D. Saumon, et al., ApJ 647, (2006) 552-557

[17] D. Stamatellos, \& A. P. Whitworth, MNRAS 392, (2009) 413-427

[18] J. R. Stauffer, G. Schultz, \& J. D. Kirkpatrick, 499, (1998) L199

[19] G. Torres, PASP 111, (1999) 169-176

[20] M. R. Zapatero Osorio, B. F. Lane, Y. Pavlenko, E. L. Martín, M. Britton, \& S. R. Kulkarni, ApJ 615, (2004) 958-971 